\title{
AN EXTREMAL PROBLEM FOR POLYNOMIALS WITH A PRESCRIBED ZERO. II
}

\section{Q. I. RAHMAN AND G. SCHMEISSER}

\begin{abstract}
Improving upon an earlier estimate it is shown that if $p_{n}(z)$ is a polynomial of degree at most $n$ such that $p_{n}(1)=0$ and $\max _{|z|-1}\left|p_{n}(z)\right|<1$, then $\left|p_{n}(0)\right|<1-(1.03369) / n+O\left(1 / n^{2}\right)$.
\end{abstract}

Let $\mathscr{P}_{n}$ be the set of all polynomials $p_{n}(z)$ of degree at most $n$ satisfying $p_{n}(1)=0$ and $\max _{|z|=1}\left|p_{n}(z)\right| \leqslant 1$. The problem of determining

$$
\mu(n):=\sup _{p_{n}(z) \in \mathscr{P}_{n}}\left|p_{n}(0)\right|
$$

was raised in [3, p. 364, Problem 8.2]. Its solution would have applications in the power sum theory of P. Turán.

It is known that there exist positive constants $c_{1}, c_{2}$ such that

$$
1-c_{1} / n \leqslant \mu(n) \leqslant 1-c_{2} / n
$$

hence for large $n$ there is a best possible estimate

$$
\mu(n) \leqslant 1-c / n+o(1 / n)
$$

where

is an absolute constant.

$$
c=\underset{n \rightarrow \infty}{\lim } n \cdot(1-\mu(n))
$$

The first upper estimate which appeared in print [5] was

$$
c \leqslant \frac{1}{\pi} \int_{-\infty}^{\infty}\left|\log \left(1-\frac{\sin ^{2} u}{u^{2}}\right)\right| d u=2.55132 \ldots
$$

Subsequently we showed in [4] that

$$
c \leqslant \pi^{2} / 8=1.23370 \ldots
$$

Various ways of arriving at a lower estimate for $c$ are known, but none of them leads to anything better than $c \geqslant 1$. For example, if $p_{n}(z) \in \mathscr{P}_{n}$, then by an inequality of Callahan [1]

$$
\left(\frac{1}{2 \pi} \int_{0}^{2 \pi}\left|p_{n}\left(e^{i \theta}\right)\right|^{2} d \theta\right)^{1 / 2} \leqslant\left(\frac{n}{n+1}\right)^{1 / 2},
$$

Received by the editors October 16, 1977 and, in revised form, May 30, 1978. AMS (MOS) subject classifications (1970). Primary 30A06; Secondary 26A82. Key words and phrases. Extremal problem, polynomials with a prescribed zero. 
whereas by a result in [2, Corollary 3]

$$
\left|p_{n}(0)\right|<\left(\frac{n}{n+1}\right)^{1 / 2}\left(\frac{1}{2 \pi} \int_{0}^{2 \pi}\left|p_{n}\left(e^{i \theta}\right)\right|^{2} d \theta\right)^{1 / 2},
$$

so that

$$
\mu(n) \leqslant \frac{n}{n+1}=1-\frac{1}{n}+O\left(\frac{1}{n^{2}}\right) .
$$

We would arrive at the same estimate if we used the formula

$$
p_{n}(0)=\frac{1}{n+1} \sum_{\nu=0}^{n} p_{n}\left(e^{i 2 \pi \nu /(n+1)}\right)
$$

and took into account the fact that the term corresponding to $\nu=0$ vanishes. For this reason several people were inclined to believe that $c$ in (2) may in fact be equal to 1 . The purpose of this paper is to show that $c$ is certainly larger than 1. Indeed, we have the following

THEOREM. For the constant $c$ in (2) the lower estimate $c>1.03369 \ldots$ holds.

Proof. It is easily seen that there exists a polynomial $p_{n}^{*}(z) \in \mathscr{P}_{n}$ such that $\mu(n)=p_{n}^{*}(0)>0$.

Now let $l_{\nu}(z)$ be the fundamental functions of Lagrange interpolation with respect to the points $e^{i \theta_{r}}$ where

$$
\theta_{\nu}:=\frac{2 \pi \nu}{n+1} \quad(\nu=0,1,2, \ldots, n),
$$

i.e.

$$
l_{0}(z)=\frac{1}{n+1} \frac{z^{n+1}-1}{z-1}, \quad l_{\nu}(z)=l_{0}\left(e^{-i \theta_{\nu} z}\right) \quad(\nu=1,2, \ldots, n) .
$$

Then $p_{n}^{*}(z)$ may be represented as

$$
p_{n}^{*}(z)=\sum_{\nu=0}^{n} p_{n}^{*}\left(e^{i \theta_{\nu}}\right) l_{\nu}(z) .
$$

Now let us write $p_{n}^{*}(z)$ in the form

$$
p_{n}^{*}(z)=q_{n}(z)+r_{n}(z),
$$

where

$$
\begin{aligned}
q_{n}(z):=\sum_{\nu=1}^{n} l_{\nu}(z) & =1-\frac{1}{n+1} \frac{z^{n+1}-1}{z-1}, \\
r_{n}(z)= & \sum_{\nu=1}^{n} \delta_{\nu} l_{\nu}(z),
\end{aligned}
$$

and

$$
\delta_{\nu}=p_{n}^{*}\left(e^{i \theta_{\nu}}\right)-1
$$


Next we observe that

$$
t(\theta):=\sum_{\nu=0}^{n}\left|l_{\nu}\left(e^{i \theta}\right)\right|^{2} \equiv 1
$$

In fact, $t(\theta)$ is a trigonometric polynomial of degree at most $n$ which, because of (4), has a period of length $2 \pi /(n+1)$. Considering the Fourier series development of $t(\theta /(n+1))$ we readily see that $t(\theta)$ must be a constant, and hence $t(\theta) \equiv t(0)=1$.

Using the Cauchy-Schwarz inequality in conjunction with (7) we obtain

$$
\max _{0<\theta<2 \pi}\left|r_{n}\left(e^{i \theta}\right)\right| \leqslant\left(\sum_{\nu=1}^{n}\left|\delta_{\nu}\right|^{2}\right)^{1 / 2}
$$

Thus, from (5) we get

$$
1 \geqslant \max _{|z|=1}\left|p_{n}^{*}(z)\right| \geqslant \max _{|z|=1}\left|q_{n}(z)\right|-\left(\sum_{\nu=1}^{n}\left|\delta_{\nu}\right|^{2}\right)^{1 / 2},
$$

and consequently

$$
\left(\sum_{\nu=1}^{n}\left|\delta_{\nu}\right|^{2}\right)^{1 / 2} \geqslant \max _{|z|=1}\left|q_{n}(z)\right|-1 .
$$

By a simple geometric consideration we deduce from (6) that

$$
\operatorname{Re} p_{n}^{*}\left(e^{i \theta_{\nu}}\right) \leqslant 1-\left|\delta_{\nu}\right|^{2} / 2 \text {. }
$$

Hence (3) and (8) give us

$$
\begin{aligned}
\mu(n) & =p_{n}^{*}(0)=\frac{1}{n+1} \sum_{\nu=0}^{n} \operatorname{Re} p_{n}^{*}\left(e^{i \theta_{\nu}}\right) \leqslant \frac{1}{n+1} \sum_{\nu=1}^{n}\left(1-\frac{\left|\delta_{\nu}\right|^{2}}{2}\right) \\
& \leqslant 1-\frac{1}{n}-\frac{1}{2 n}\left(\max _{|z|=1}\left|q_{n}(z)\right|-1\right)^{2}+O\left(\frac{1}{n^{2}}\right) .
\end{aligned}
$$

Finally, taking $\alpha=4.085573885$ we obtain by a numerical calculation

$$
\begin{aligned}
\left|q_{n}\left(e^{i \alpha / n}\right)\right| & =\left\{\left(1-\frac{\sin \alpha}{\alpha}\right)^{2}+\left(\frac{1-\cos \alpha}{\alpha}\right)^{2}\right\}^{1 / 2}+O\left(\frac{1}{n}\right) \\
& =1.259590522 \ldots+O\left(\frac{1}{n}\right) .
\end{aligned}
$$

Using this value as a lower estimate for $\max _{|z|=1}\left|q_{n}(z)\right|$ in (9) we arrive at the desired result.

\section{REFERENCES}

1. F. P. Callahan, Jr., An extremal problem for polynomials, Proc. Amer. Math. Soc. 10 (1959), 754-755.

2. A. Giroux and Q. I. Rahman, Inequalities for polynomials with a prescribed zero, Trans. Amer. Math. Soc. 193 (1974), 67-98.

3. Ch. Pommerenke, Problems in complex function theory, Bull. London Math. Soc. 4 (1972), 354-366. 
4. Q. I. Rahman and G. Schmeisser, Some inequalities for polynomials with a prescribed zero, Trans. Amer. Math. Soc. 216 (1976), 91-103.

5. Q. I. Rahman and F. Stenger, An extremal problem for polynomials with a prescribed zero, Proc. Amer. Math. Soc. 43 (1974), 84-90.

Department of Mathematics and Statistics, Universtty of Montreal, Montreal, QUEBeC H3C 3J7, CANADA

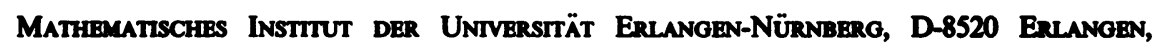
BisMARCKSTRASSE $1 \frac{1}{2}$, WeSt GERMANY 